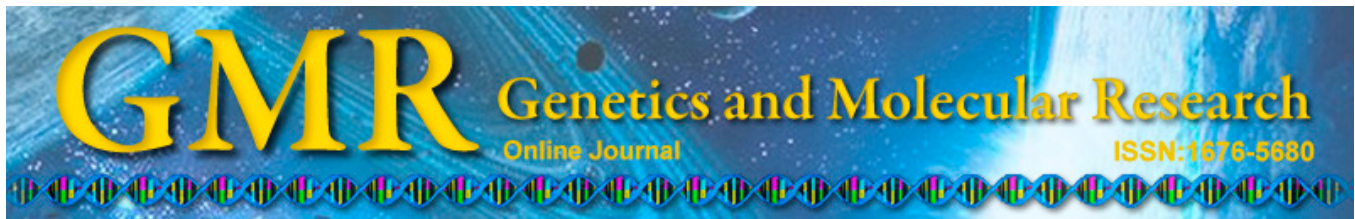

\title{
Treatment of silicosis with hepatocyte growth factor-modified autologous bone marrow stromal cells: a non-randomized study with follow-up
}

W.W. Liu ${ }^{1}$, H.X. Wang ${ }^{2}$, W. Yu ${ }^{1}$, X.Y. Bi ${ }^{3}$, J.Y. Chen ${ }^{1}$, L.Z. Chen ${ }^{1}$, L. Ding ${ }^{2}$, D.M. Han' ${ }^{2}$ Z.K. Guo ${ }^{1}$ and Y.X. Lei ${ }^{4}$

${ }^{1}$ Department of Occupational Diseases, Guangzhou No. 12 People's Hospital Affiliated to Guangzhou Medical University, Guangzhou, China

${ }^{2}$ Department of Hematology, General Hospital of the Air Force, Beijing, China ${ }^{3}$ Department of Internal Medicine, Guangzhou Development District Hospital, Guangzhou, China

${ }^{4}$ School of Public Health, Guangzhou Medical University, China

Corresponding authors: Y.X. Lei / Z.K. Guo

E-mails: gz-leizeng@163.com / Zikuanguo@aliyun.com

Genet. Mol. Res. 14 (3): 10672-10681 (2015)

Received February 20, 2015

Accepted May 8, 2015

Published September 9, 2015

DOI http://dx.doi.org/10.4238/2015.September.9.7

ABSTRACT. Pulmonary silicosis is an irreversible and untreatable disease that is characterized by interstitial lesions and perpetual fibrosis in the lungs. This study was performed to determine whether mesenchymal stem cells (MSCs) and hepatocyte growth factor (HGF) could exhibit therapeutic effects on human silicosis. This nonrandomized uncontrolled trial comprised four patients with pulmonary silicosis who had developed lung fibrosis and received autologous bone marrow MSCs previously transfected by a vector containing human HGF cDNA (MSCs/HGF). MSCs/HGF were intravenously administered weekly for three consecutive weeks at a dose of 2 $\mathrm{x} 10^{6}$ cells $/ \mathrm{kg}$. Pulmonary function, high kilo-voltage chest X-ray radiography, computed tomography (CT) scan, and peripheral blood 
lymphocyte subset and serum $\operatorname{IgG}$ concentrations were evaluated after cell therapy. The treatment was found to be generally safe. Symptoms such as cough and chest distress gradually ameliorated at six months post-therapy, accompanied by the significant improvement of pulmonary function. The ratios of the peripheral CD4- and CD8positive cell concentrations were increased $(\mathrm{P}<0.05)$. Furthermore, the serum $\operatorname{IgG}$ levels in these patients were decreased and reached the normal range $(\mathrm{P}<0.05)$. CT scans showed partial absorption of the nodular and reticulonodular lesions in the lungs during follow-up of at least 12 months. The effectiveness of this novel regimen observed in these patients suggests that a placebo-controlled clinical trial needs to be developed. This study carries trial registration No. NCT01977131 (ClinicalTrials.gov).

Key words: Pulmonary silicosis; Mesenchymal stem cells; Cytotherapy; Hepatocyte growth factor; Gene therapy

\section{INTRODUCTION}

Silicosis is a potentially fatal and irreversible disease caused by inhaling crystalline silica dust. It is characterized by interstitial lesions and, in some cases, progressive and massive fibrosis of the lungs due to the persistent bronchoalveolar inflammation elicited by the silica particles. Silicosis is a preventable but untreatable disorder; there are no effective treatments available for silicosis once the disease is contracted (Leung et al., 2012).

Mesenchymal stem cells (MSCs) are adult stem cells characterized by their differentiation capacity (fat, cartilage, and bone) and immunomodulatory function. Because of these features, they have been increasingly utilized in clinical trials as both cell replacement and immune suppression strategies. In addition, increasing numbers of reports have demonstrated that transfusion of MSCs displays beneficial effects on the management of lung fibrosis. This is thought to arise because MSCs are primarily sequestered by the pulmonary circulation early after systematic administration, and because the transfused MSCs preferentially home to or are implanted into the lungs in response to the local inflammation environment (Jones and Rankin, 2011; Ricciardi et al., 2012; Hori et al., 2013). Exogenous MSCs might secrete a variety of bioreactive substances to inhibit the production of transforming growth factor-beta and reduce inflammation and collagen deposition (Kotton et al., 2001). MSCs might also adopt an epithelium-like phenotype to take a direct part in the reparative progress of the damaged lung tissues (Kotton et al., 2001; Krause et al., 2001). Furthermore, in bleomycin-induced fibrosis, loss of resident MSCs in the lungs might contribute to disease development (Jun et al., 2011). These investigations support the application of MSCs in the treatment of silicosis.

Hepatocyte growth factor (HGF) is a well-recognized factor that has the capacity to protect against fibrosis probably by promoting cell proliferation and survival, preventing the epithelial-mesenchymal transition, and by -its pro-angiogenic activity (Chakraborty et al., 2013). Our previous research has shown that HGF-modified MSCs (MSCs/HGF), generated by the transfection of an HGF cDNA-containing vector into MSCs, exhibit potent immunosuppressive activity (Bian et al., 2009). In patients with standard interstitial pneumonia, HGF was found to be expressed in the MSCs primarily located in fibrotic areas close to the 
hyperplastic alveolar epithelium (Gazdhar et al., 2013). Furthermore, HGF-transfected bone marrow MSCs could reduce bleomycin-induced lung fibrosis, suggesting that MSCs/HGF have antifibrotic properties (Gazdhar et al., 2013). MSCs/HGF could be sequestered in the radiation-injured lungs, thereby reducing the secretion and expression of pro-inflammatory cytokines and increasing the expression of the anti-inflammatory cytokine IL-10 (Wang et al., 2013). Based on such reports, we postulated that intravenous injection of MSCs/HGF might have beneficial effects on the amelioration of the progressive fibrosis caused by silicosis. In this study, we report the therapeutic outcome of the administration of bone marrow MSCs modified with the HGF gene in patients with pulmonary silicosis.

\section{MATERIAL AND METHODS}

\section{Patients}

Patients were involved in this study from January 2009 to June 2010 and were followed up from June 2009 to June 2011. All patients were diagnosed with silicosis according to the Diagnosis Criteria for Silicosis (No. GBZ70-2009) released by the People's Republic of China. All subjects provided written informed consent before entering the study. The study was approved by the Ethics Committee and Institution Review Board of the Twelfth Hospital, Guangzhou, China. This clinical trial was registered as NCT01977131 on Nov. 05, 2013 at $\mathrm{http} / / /$ clinicaltrials.gov/. This study was not registered prior to initiation of participant enrollment as the clinical trial registration system in China. Therefore, Chinese researchers have deemed that registrations might be carried out at any time.

\section{Treatment protocols prior to cell therapy}

The patients received at least one-month of combined treatment protocols including expectorants (acetylcysteine and ambroxol hydrochloride), the immune-regulation agent pidotimod, and a macrophage-protective agent, polyvinylpyridine-n-oxide. In addition, bronchoalveolar lavage with $0.9 \%$ saline containing dexamethasone was also performed. No significant improvements were achieved from these treatments.

\section{Determination of serum ceruloplasmin and IgG concentrations}

The serum concentration of ceruloplasmin, a NO oxidase that can serve as a marker of alveolar-capillary permeability in the progression of pulmonary silicosis (Zhicheng et al., 1986; Baker et al., 2000; Shiva et al., 2006), was determined using the immuno-turbidometric method. The serum concentration of IgG was also assessed using this technique.

\section{Lung function testing}

The parameters of lung function were evaluated with a pneumonometer (CHEST GRAPH HI-701, Tokyo, Japan). The determined and estimated forced vital capacity (FVC), the volume that has been exhaled at the end of the first second of forced expiration (FEV1), and the one second rate (FEV1/FVC\%) were documented. Additionally, arterial blood oxy- 
hemoglobin saturation was determined with an IL-1400 blood gas and electrolyte analyzer (Instrumentation Laboratory, MA, USA).

\section{Preparation of MSCs and MSCs/HGF}

After obtaining approval from the Ethics Committee and Institution Review Board of the Twelfth Hospital and written informed consent from the patient, $30-50 \mathrm{~mL}$ bone marrow aspirate was harvested from the posterior iliac crest of the patient. MSC cultures were routinely taken. Briefly, marrow mononucleated cells including those from the collagenase type I-digested debris were collected by gradient density centrifugation. The cells were suspended in alpha-MEM supplemented with 10\% fetal serum derived from lots (STEMCELL Technologies, Vancouver, BC, Canada) and seeded into two $150 \mathrm{~mm}$ culture dishes. MSCs at passage two were harvested and split into three samples. One sample was used for flow cytometric analysis as described below. Another was passaged and cryopreserved for further utilization. The third sample was cultured until the confluence reached approximately $80 \%$, followed by transfection with plasmid HGF (kindly provided by Dr. Wu Zuze) using Lipofectamine 2000 agents (Invitrogen, Carlsbad, CA, USA). The transfected culture was further maintained for $48 \mathrm{~h}$ before cell harvesting.

\section{Cell harvesting and injection}

Two days before cell harvesting, aliquots of the culture medium $(500 \mu \mathrm{L})$ were collected for a rapid exclusion screen of bacterium and fungus contamination using an MB-80 Microbiology Kinetic Rapid Reader (Beijing Gold Mountainriver Development Co., Beijing, China). MSCs/HGF were harvested by trypsin digestion, washed twice in saline and counted with a trypan blue exclusion test. The cells were then suspended in $100 \mathrm{~mL}$ saline containing $10 \mathrm{~mL} 10 \%$ human plasma albumin for injection. Within $1 \mathrm{~h}$ after collection, MSCs/HGF were intravenously infused into the patient at a dose of $2 \times 10^{6} \mathrm{cells} / \mathrm{kg}$ over approximately $30 \mathrm{~min}$. MSC injections were then performed weekly for an additional two weeks. All patients were monitored closely during and within $24 \mathrm{~h}$ of cell injection. Body temperature, blood pressure, pulse, respiratory rate, and electrocardiogram were monitored.

\section{Flow cytometric analysis}

The collected cells at passage 2 were reacted with PE-conjugated mouse monoclonal antibodies against human CD29, CD31, CD44, CD45, and CD73 (Becton Dickinson, Bedford, MA, USA) for $30 \mathrm{~min}$ in the dark. After washing twice in phosphate buffered saline (PBS), the cells were detected using a flow cytometer and the detection events were analyzed with WinMdi-29 (http://facs.scripps.edu/software/WinMDI29.msi). In addition, heparinized peripheral blood was drawn before and six months after cell therapy and the proportions of $\mathrm{CD} 4^{+}$and $\mathrm{CD} 8^{+}$cells in the peripheral blood were assessed by flow cytometry (BD Biosciences, San Jose, CA, USA).

\section{Statistical analysis}

The data are reported as means \pm standard deviation. The Student $t$ test was used to analyze the difference among the data. All the analyses were carried out using SPSS17.0 software (SPSS, Chicago, IL, USA). P values $<0.05$ were considered to be statistically significant. 


\section{RESULTS}

\section{Study patients}

We enrolled 48 patients diagnosed with pulmonary silicosis using the Silicosis Diagnosis Criteria in China from January 2009 to June 2010; patients were followed up from June 2009 to June 2011. Of the eligible 48 patients, 44 did not meet inclusion criteria (4), or were not consented for protocol screening tests or were ineligible for other reasons (40). Ultimately, four patients were consented for protocol screening tests. The characteristics of the enrolled patients are summarized in Table 1. The mean age was $41.5 \pm 6.6$ years and the mean silica dust contact time was $9 \pm 6.2$ years before they was diagnosed with pulmonary silicosis and admitted into the Guangzhou Twelfth People's Hospital, China. All patients had complaints of recurrent cough and chest distress for at least one month. Three of the patients used to work in the sand blasting industry and one in mine blasting. High kilovoltage chest X-rays and thinsection chest CT scans were used to identify lesions in the lungs. Infections by tuberculosis bacteria, herpes simplex virus, cytomegalovirus, rubella virus, and toxoplasm were excluded by serum antibody detection. All patients were treated with MGCs/HGF. A consort style flowchart of participants through the study is shown in Figure 1.

Table 1. Patient characteristicsof Silicosis.
\begin{tabular}{llccccc}
\hline Cases & Gender & Age & Dust-contact time (year) & Silicosis stages* & Disease course & Types of job \\
\hline 1 & M & 37 & 8 & II & $1 \mathrm{y}$ & Sand blasting \\
2 & F & 41 & 5 & I & $3 \mathrm{~m}$ & Sand blasting \\
3 & F & 37 & 5 & II & $1 \mathrm{~m}$ & Sand blasting \\
4 & M & 51 & 18 & I & $1 \mathrm{~m}$ & Mine blasting \\
\hline
\end{tabular}

*Silicosis is divided into four stages (observed, I, II, III) according to seriously levels with Diagnosis Criteria for Silicosis (No. GBZ70-2009) of China.

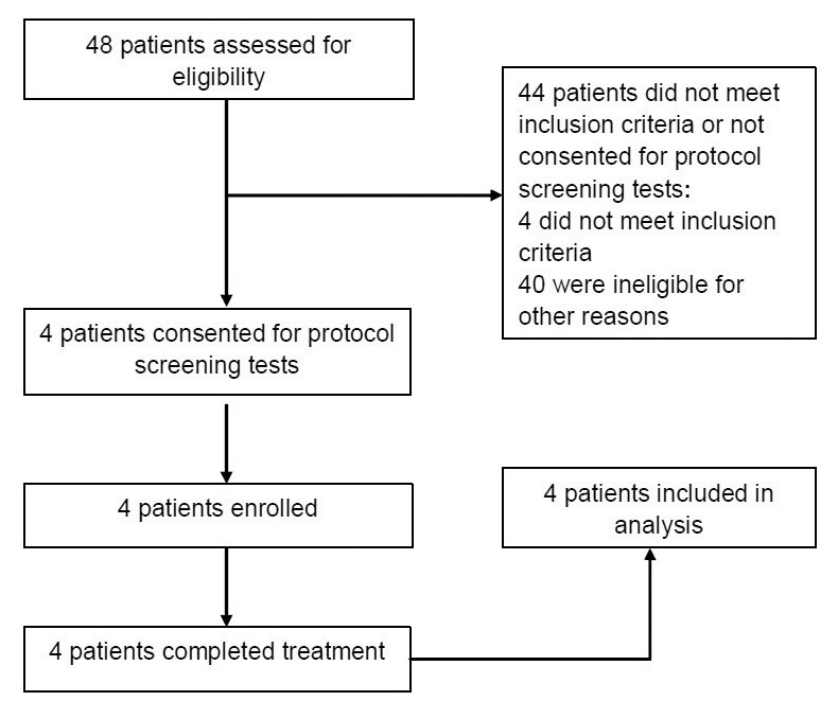

Figure 1. Consort style flowchart of participants through the study. 


\section{MSC quality control}

MSCs were isolated from bone marrow samples of the patients with silicosis and the adherent cells at passage 2 were evaluated. Flow cytometric analysis showed that the adherent cells were homogenously positive for CD29, CD44, and CD73 and negative for CD31 and CD45 (not shown), indicating the typical surface marker expression profiles of bone marrow MSCs. The cells met the minimal criteria of MSCs stated by the International Society for Cellular Therapy (Dominici et al., 2006). In addition, the results from the microbiology reader showed that the culture media collected prior to cell harvesting were negative for fungal and bacterial contamination, according to the concentrations measured of (1-3)- $\beta$-D-glucan and endotoxin, respectively.

\section{Acute toxicities}

The therapy was generally found to be safe. Two patients (Nos. 1 and 3) had fever and chilling about 30 min after cell injection. The symptoms were relieved after they received $10 \mathrm{mg}$ dexamethasone by intravenous injection. No fever, headache, diarrhea or vomiting were observed in the other two patients. Routine monitoring found no evidence of cardiac toxicity.

\section{Clinical follow-up}

Pulmonary function tests showed that the FVC and FEV1 measures marginally increased in the patients 6 months post-transfusion, but the FVC and FEV1 averages were not significantly different before and after treatment (Table 2). In addition, the arterial blood oxyhemoglobin saturation $(\mathrm{SpO} 2)$ was significantly enhanced $(\mathrm{P}<0.05)$, consistent with the partial relief or even disappearance of symptoms including cough and dyspnea. During the 12-month follow-up for each patient, thin-section CT indicated that in one patient (No. 3), the nodular lesions had been partially absorbed (Figure 2), which was further supported by chest high kilo-voltage radiography (Figure 3). Follow-up of patient No. 4 with chest CT scan also showed partial lesion absorption, but no significant improvements were observed in other two patients (Nos. 1 and 2) (Figure 4).

Table 2. Pulmonary function testing before and 6 months after cell therapy in the cases of silicosis.

\begin{tabular}{|c|c|c|c|c|c|c|c|}
\hline \multirow[t]{2}{*}{ Cases } & \multirow[t]{2}{*}{ Treatment } & \multicolumn{2}{|c|}{$\mathrm{FVC} \%$} & \multicolumn{2}{|c|}{ FEV1\% } & \multicolumn{2}{|c|}{$\mathrm{SpO} 2$} \\
\hline & & Baseline & Post & Baseline & Post & Baseline & Post \\
\hline 1 & MSCs/HGF & 89.5 & 91.8 & 85.2 & 87.9 & 87.7 & 92.2 \\
\hline 2 & MSCs/HGF & 94.1 & 96.8 & 97.4 & 94.1 & 81.2 & 99.0 \\
\hline 3 & MSCs/HGF & 67.0 & 64.6 & 70.3 & 82.0 & 87.4 & 99.0 \\
\hline 6 & MSCs/HGF & 75.6 & 85.2 & 71.8 & 88.5 & 90.2 & 99.0 \\
\hline Mean & & 81.6 & 84.6 & 81.2 & 88.1 & 86.6 & 97.3 \\
\hline$P$ value & & \multicolumn{2}{|c|}{$>0.05$} & \multicolumn{2}{|c|}{$>0.05$} & \multicolumn{2}{|c|}{$<0.05$} \\
\hline
\end{tabular}

Data are reported as means \pm standard deviation. Paired $t$ test was used to analyze the difference between the date before and 6 months after cell therapy, $\mathrm{P}<0.05$ means statistically significant. 


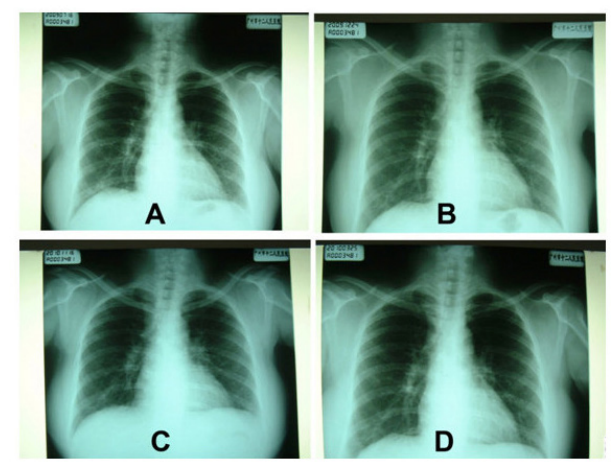

Figure 2. Chest high kilovoltage X-ray radiography findings after cellular treatment. Partial lesion absorption is observed in the lower-left lung tissue. The patient No. 3 in this group was diagnosed as silicosis stage II and received MSCs/HGF. A. Pretreatment; B. 5 months after cell therapy; C. one year after treatment; D. 16 months post treatment.

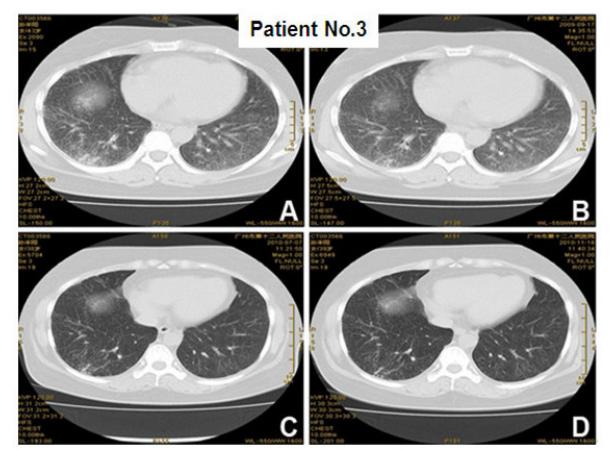

Figure 3. Chest CT scan findings during the follow-up of patient, No. 3. A. Pretreatment; B. 5 months after cell treatment; C. 12 months post-therapy; D. 16 months post-therapy. Partial lesion absorption in the lower-left lung tissue is evident.

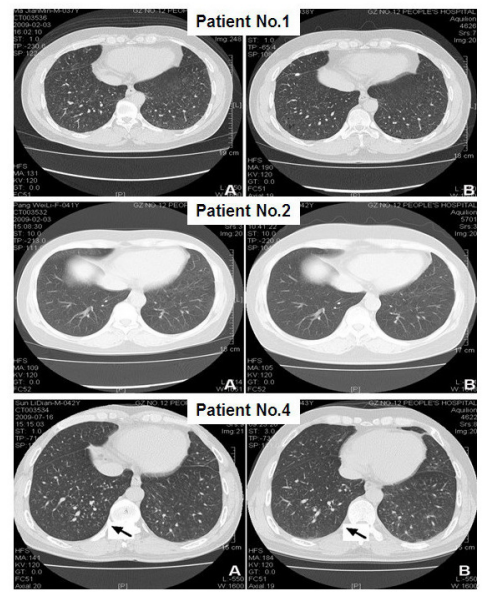

Figure 4. Follow-up of patients Nos. 1, 2, 4 with chest CT scan. A. Pre-treatment; B. one year post cell therapy. The patient No. 4 with chest CT scan showed the partial lesion absorption (arrow), but no obvious lesion absorption was observed in patients Nos. 1 and 2. 


\section{Serum IgG and peripheral CD4/CD8 ratio}

To observe the potential therapeutic mechanisms underlying MSC therapy, the serum IgG level and peripheral $\mathrm{T}$ lymphocyte subsets from the patients were evaluated six months after administering MSCs. The results showed that six months after MSC therapy, the ratios of peripheral blood $\mathrm{CD}^{+} / \mathrm{CD}^{+}$cell concentrations were greatly enhanced $(\mathrm{P}<0.05)$. The $\mathrm{CD}^{+} /$ $\mathrm{CD}^{+}$ratio was evidently restored in patient No. 1 after MSC therapy. Furthermore, the serum IgG levels in these patients were decreased to within the normal range $(7.6-16.6 \mathrm{~g} / \mathrm{L})(\mathrm{P}<$ 0.05 ) (Table 3). Ceruloplasmin is a multicopper oxidase with $\mathrm{NO}$ oxidase and nitrite synthase activities (Shiva et al., 2006); the serum ceruloplasmin level is recognized as not only a marker of pulmonary infection (Valletta et al., 1992; Cernat et al., 2011) but also as a predictor of collagen deposition in the injured lungs (Sauni et al., 2012). As shown in Table 3, the average ceruloplasmin level in the patients was $0.25 \mathrm{~g} / \mathrm{L}$ (normal range: $0.2-0.6 \mathrm{~g} / \mathrm{L}$ ) at six months after MSC therapy, slightly lower than that prior to treatment $(0.28 \mathrm{~g} / \mathrm{L})$. These results suggest that MSCs/HGF might exert a therapeutic effect by inhibiting the chronic inflammation reaction in the silicified lungs.

Table 3. Immune-associated parameters before and after cell therapy in the cases of silicosis.

\begin{tabular}{|c|c|c|c|c|c|c|}
\hline \multirow[t]{2}{*}{ Cases } & \multicolumn{2}{|c|}{$\mathrm{CD} 4 / \mathrm{CD} 8$ ratio } & \multicolumn{2}{|c|}{ Serum IgG (g/L) } & \multicolumn{2}{|c|}{ Ceruloplasmin $(\mathrm{g} / \mathrm{L})$} \\
\hline & Baseline & Post & Baseline & Post & Baseline & Post \\
\hline 1 & 0.86 & 1.45 & 21.19 & 15.74 & 0.20 & 0.26 \\
\hline 2 & 1.57 & 2.00 & 10.29 & 9.03 & 0.40 & 0.19 \\
\hline 3 & 1.59 & 1.89 & 16.10 & 12.99 & 0.31 & 0.31 \\
\hline 4 & 1.45 & 1.88 & 15.61 & 12.35 & 0.21 & 0.22 \\
\hline Mean & 1.37 & 1.81 & 15.79 & 12.53 & 0.28 & 0.25 \\
\hline$P$ value & \multicolumn{2}{|c|}{$<0.05$} & \multicolumn{2}{|c|}{$<0.05$} & \multicolumn{2}{|c|}{$>0.05$} \\
\hline
\end{tabular}

Data are reported as means \pm standard deviation. Paired $t$ test was used to analyze the difference between baseline and post-therapy in the cases of silicosis, $\mathrm{P}<0.05$ means statistically significant.

\section{DISCUSSION}

Until recently, lung transplantation has been the only modality to cure silicosis. However, this strategy is strictly limited by a dearth of donors and serious graft rejection. In this preliminary report, a new treatment protocol was designed based on the retention of MSCs in the lungs and the paracrine secretion activities of transplanted MSCs. The results presented here indicate that intravenous injection of bone marrow MSCs is generally safe and might exert therapeutic activity in some patients.

Pulmonary silicosis is an uncontrollable progressive disease that is characterized by aggravation of restrictive ventilatory dysfunction. In this group, the pulmonary function testing showed that the increase in FVC and FEV1 in the six months after cell therapy was only marginal, suggesting that restrictive ventilatory dysfunction caused by fibrosis was not dramatically improved by MSC/HGF therapy.

Arterial blood oxyhemoglobin saturation was significantly enhanced, accompanied by partial relief of symptoms such as cough and dyspnea. The results indicate that anatomically structural impairment in the lungs caused by the permanent residue of silica dust could not be repaired by the MSCs or the MSCs/HGF treatment described here. MSC therapy seemingly 
improved gas exchange between the alveoli-pulmonary blood circulation, probably by reducing the severity of the inflammation reaction elicited by the silica dust.

Recent studies have demonstrated that silicosis is an immune-associated disease as pulmonary silicosis is usually accompanied by abnormalities of immune parameters, and the patients with silicosis have a tendency to suffer from autoimmune diseases (Valletta et al., 1992; Cooper et al., 2002; Carlsten et al., 2007; Sauni et al., 2012). In this study, we found that IgG levels were elevated in patients with silicosis, and that this elevation could be downregulated by MSC therapy. Increased IgG levels were also reported by Sauni et al., 2012. In a chronic asthmatic mouse model, it was found that administering MSCs could decrease airway inflammation and airway remodeling by elevating IL-12 concentrations and the number of regulatory $\mathrm{T}$ cells, resulting in the re-balance of the local immune system (Ge et al., 2013).

Furthermore, CT scans and chest X-rays demonstrated the disappearance of some silica nodules in two patients (Nos. 3 and 4) after MSC therapy, suggesting that MSCs/HGF could give rise to structural healing from the silicosis. However, no definite conclusion could be drawn to fully elucidate the beneficial effects of MSCs/HGF.

In conclusion, our results suggest that MSC/HGF administration is safe and effective in some patients for the treatment of silicosis. A placebo-controlled clinical trial with a larger number of participants should be developed to draw a concrete conclusion regarding the effectiveness and the long-term safety of this treatment.

\section{Conflicts of interest}

The authors declare no conflict of interest.

\section{ACKNOWLEDGMENTS}

Research supported by the Guangzhou Key Projects of Medical Science and Technology, China (\#2008-ZDi-04 and \#201102A212004), a Science and Technology Planning Project of Guangdong Province, China (\#2010B031600019), and the National Natural Science Foundation of China (\#81373038 to L.Y.). We would like to acknowledge the excellent work of all dedicated research staff and medical students in the project.

\section{REFERENCES}

Baker CS, Evans TW and Haslam PL (2000). Measurement of ceruloplasmin in the lungs of patients with acute respiratory distress syndrome: is plasma or local production the major source? Respiration 67: 533-538.

Bian L, Guo ZK, Wang HX, Wang JS, et al. (2009). In vitro and in vivo immunosuppressive characteristics of hepatocyte growth factor-modified murine mesenchymal stem cells. In Vivo 23: 21-27.

Carlsten C, De Roos AJ, Kaufman JD, Checkoway H, et al. (2007). Cell markers, cytokines, and immune parameters in cement mason apprentices. Arthritis Rheum. 57: 147-153.

Cernat RI, Mihaescu T, Vornicu M, Vione D, et al. (2011). Serum trace metal and ceruloplasmin variability in individuals treated for pulmonary tuberculosis. Int. J. Tuberc. Lung Dis. 15: 1239-1245, i.

Chakraborty S, Chopra P, Hak A, Dastidar SG, et al. (2013). Hepatocyte growth factor is an attractive target for the treatment of pulmonary fibrosis. Expert Opin. Investig. Drugs 22: 499-515.

Cooper GS, Miller FW and Germolec DR (2002). Occupational exposures and autoimmune diseases. Int. Immunopharmacol. 2: 303-313.

Dominici M, Le Blanc K, Mueller I, Slaper-Cortenbach I, et al. (2006). Minimal criteria for defining multipotent mesenchymal stromal cells. The International Society for Cellular Therapy position statement. Cytotherapy 8: 315-317. 
Gazdhar A, Susuri N, Hostettler K, Gugger M, et al. (2013). HGF expressing stem cells in usual interstitial pneumonia originate from the bone marrow and are antifibrotic. PLoS One 8: e65453.

Ge X, Bai C, Yang J, Lou G, et al. (2013). Effect of mesenchymal stem cells on inhibiting airway remodeling and airway inflammation in chronic asthma. J. Cell Biochem. 114: 1595-1605.

Hori M, Juniantito V, Izawa T, Ichikawa C, et al. (2013). Distribution of cells labelled by a novel somatic stem cellrecognizing antibody (A3) in pulmonary genesis and bleomycin induced pulmonary fibrosis in rats. J. Comp. Pathol. 148: 385-395.

Jones CP and Rankin SM (2011). Bone marrow-derived stem cells and respiratory disease. Chest 140: 205-211.

Jun D, Garat C, West J, Thorn N, et al. (2011). The pathology of bleomycin-induced fibrosis is associated with loss of resident lung mesenchymal stem cells that regulate effector T-cell proliferation. Stem Cells 29: 725-735.

Kotton DN, Ma BY, Cardoso WV, Sanderson EA, et al. (2001). Bone marrow-derived cells as progenitors of lung alveolar epithelium. Development 128: 5181-5188.

Krause DS, Theise ND, Collector MI, Henegariu O, et al. (2001). Multi-organ, multi-lineage engraftment by a single bone marrow-derived stem cell. Cell 105: 369-377.

Leung CC, Yu IT and Chen W (2012). Silicosis. Lancet 379: 2008-2018.

Ricciardi M, Malpeli G, Bifari F, Bassi G, et al. (2012). Comparison of epithelial differentiation and immune regulatory properties of mesenchymal stromal cells derived from human lung and bone marrow. PLoS One 7: e35639.

Sauni R, Oksa P, Lehtimaki L, Toivio P, et al. (2012). Increased alveolar nitric oxide and systemic inflammation markers in silica-exposed workers. Occup. Environ. Med. 69: 256-260.

Shiva S, Wang X, Ringwood LA, Xu X, et al. (2006). Ceruloplasmin is a NO oxidase and nitrite synthase that determines endocrine NO homeostasis. Nat. Chem. Biol. 2: 486-493.

Valletta EA, Rigo A, Bonazzi L, Zanolla L, et al. (1992). Modification of some markers of inflammation during treatment for acute respiratory exacerbation in cystic fibrosis. Acta Paediatr. 81: 227-230.

Wang H, Yang YF, Zhao L, Xiao FJ, et al. (2013). Hepatocyte growth factor gene-modified mesenchymal stem cells reduce radiation-induced lung injury. Hum. Gene Ther. 24: 343-353.

Zhicheng S, Zhiming Y, Lata A and Yuhua H (1986). Serum angiotensin converting enzyme, ceruloplasmin, and lactic dehydrogenase in anthracosilicosis and anthracosilicotuberculosis. Br. J. Ind. Med. 43: 642-643. 